Article

\title{
Active Expression of Membrane-Bound L-Amino Acid Deaminase from Proteus mirabilis in Recombinant Escherichia coli by Fusion with Maltose-Binding Protein for Enhanced Catalytic Performance
}

\author{
Dan-Ping Zhang ${ }^{1}$, Xiao-Ran Jing ${ }^{1}$, An-Wen Fan ${ }^{1}$, Huan Liu ${ }^{1}$, Yao Nie ${ }^{1,2, * \mathbb{C}}$ and Yan Xu ${ }^{1,3}$ \\ 1 School of Biotechnology and Key laboratory of Industrial Biotechnology, Ministry of Education, Jiangnan \\ University, Wuxi 214122, China; danielpingzhang@163.com (D.-P.Z.); rushjxr794@163.com (X.-R.J.); \\ fananwen1998@163.com (A.-W.F); 18635924851@163.com (H.L.); yxu@jiangnan.edu.cn (Y.X.) \\ 2 Suqian Industrial Technology Research Institute of Jiangnan University, Suqian 223814, China \\ 3 State Key Laboratory of Food Science and Technology, Jiangnan University, Wuxi 214122, China \\ * Correspondence: ynie@jiangnan.edu.cn; Tel.: +86-510-8519-7760
}

Received: 24 December 2019; Accepted: 6 February 2020; Published: 10 February 2020

\begin{abstract}
L-amino acid deaminases (LAADs) are membrane flavoenzymes that catalyze the deamination of neutral and aromatic L-amino acids to $\alpha$-keto acids and ammonia. LAADs can be used to develop many important biotechnological applications. However, the transmembrane $\alpha$-helix of LAADs restricts its soluble active expression and purification from a heterologous host, such as Escherichia coli. Herein, through fusion with the maltose-binding protein (MBP) tag, the recombinant E. coli BL21 (DE3)/pET-21b-MBP-PmLAAD was constructed and the LAAD from Proteus mirabilis (PmLAAD) was actively expressed as a soluble protein. After purification, the purified MBP-PmLAAD was obtained. Then, the catalytic activity of the MBP-PmLAAD fusion protein was determined and compared with the non-fused PmLAAD. After fusion with the MBP-tag, the catalytic efficiency of the MBP-PmLAAD cell lysate was much higher than that of the membrane-bound PmLAAD whole cells. The soluble MBP-PmLAAD cell lysate catalyzed the conversion of $100 \mathrm{mM}$ L-phenylalanine (L-Phe) to phenylpyruvic acid (PPA) with a 100\% yield in $6 \mathrm{~h}$. Therefore, the fusion of the MBP-tag not only improved the soluble expression of the PmLAAD membrane-bound protein, but also increased its catalytic performance.
\end{abstract}

Keywords: L-amino acid deaminases; membrane-bound protein; fusion protein; soluble expression; maltose-binding protein tag

\section{Introduction}

L-amino acid deaminases (LAADs; EC 1.4.3.2) belong to a family of amino acid dehydrogenases that catalyze the formation of $\alpha$-keto acid from L-amino acid and release ammonium and $\mathrm{H}_{2} \mathrm{O}$ as well [1-4]. LAADs have been identified in several bacterial genera including Proteus, Providencia, and Morganella [2,5]. Some bacteria, such as Proteus mirabilis, express two types of LAAD which have similar sequences but have distinct substrate preferences: type I prefers aliphatic and aromatic amino acids, while type II shows significant activity with basic amino acids such as histidine and arginine [2,3,6-9]. Both types of LAAD contain a single membrane-spanning helix, which is anchored to the cytomembrane surface through the N-terminal transmembrane helix [2,9].

Recently, membrane-bound LAADs have been the focus of studies due to their potential biotechnological applications in industry [8-11]. A series of LAAD-based technologies has been developed to efficiently transform L-amino acids to $\alpha$-keto acids such as ketoglutaric acid, 
methylthiobutyric acid, and phenylpyruvic acid (PPA) in an eco-friendly manner [3,7,12-16]. Among them, PPA is an important multi-functional organic acid which is widely used in the pharmaceutical, food, and chemical industries [6,17]. LAADs are also used to produce pure D-amino acids by removing L-amino acids from racemic mixtures [18-21]. Other potential applications, such as their use as biosensors of L-amino acid concentration in the medical and food industries, have also been proposed [22-24].

As the potential applications of LAADs have increased, many researchers have focused on the study of membrane-bound LAADs. Baek et al. cloned, expressed, and characterized a type II L-amino acid deaminase from P. mirabilis KCTC $2566[2,14]$. To facilitate the purification of a membrane-bound LAAD from P. mirabilis, Liu et al. removed the $\mathrm{N}$-terminal transmembrane region (from 21 to 87 nucleotides) from the enzyme to block its binding to the membrane [3,6,10]. Similarly, to study the crystal structure of a membrane-bound LAAD from P. vulgaris (PvLAAD), Ju et al. also removed the N-terminal transmembrane region of the enzyme to obtain purified PvLAAD [9]. However, the LAAD was still expressed in the form of an inclusion body. Although it seems that the LAAD whole-cell biocatalysts are more suitable for catalysis, in order to better study the applicable feasibility of the enzyme, it is necessary to improve its soluble expression.

The maltose-binding protein (MBP) is the product of the malE gene in Escherichia coli K12. The protein consists of 396 amino acids [25-30] and has a molecular weight of $40 \mathrm{KDa}$ [25,26]. The N-terminal of the MBP has a signal peptide sequence of 26 amino acids, which could guide the final expression of the MBP to locate in the periplasm [25,27]. According to the directional expression characteristics of the MBP protein, researchers constructed an MBP tag to promote the heterologous soluble expression of some proteins [27,30]. MBP has been used with a few membrane proteins and the MBP-tag has been employed for the membrane-binding protein to locate in the periplasm [25].

In this study, we fused the LAAD from P. mirabilis (PmLAAD) with the MBP-tag for soluble expression in recombinant $E$. coli. Then, the MBP-tag fused LAAD (MBP-PmLAAD) was expressed and purified as a soluble protein. After protease digestion and purification to remove the MBP-tag, the soluble purified PmLAAD was obtained. Then, the catalytic properties of the MBP-PmLAAD fusion, the soluble PmLAAD, and the MBP-PmLAAD cell lysate were studied. Finally, the catalytic efficiency of the MBP-fused LAAD and the membrane-binding LAAD were compared.

\section{Results and Discussion}

\subsection{Construction of MBP-PmLAAD Recombinant Protein Expression Plasmids}

The PCR products of PmLAAD were inserted into the vector $\mathrm{pET}-21 \mathrm{~b}$. The $1416 \mathrm{bp}$ PmLAAD (Figure 1B) open reading frame was amplified from P. mirabilis KCTC 2566. The MBP-PmLAAD vector, designed to produce PmLAAD proteins in the cytoplasm, was constructed by fusing the PmLAAD gene to the $M B P$ gene driven by a T7 promoter (Figure $1 \mathrm{~A}$ ). The MBP was generally used to improve the solubility of the fusion proteins in the E. coli system and expressed at the N-end of heterologous MBP-PmLAAD products. The vector encoding the MBP-PmLAAD products was used to transform E. coli BL21 (DE3) and produce fusion proteins. 

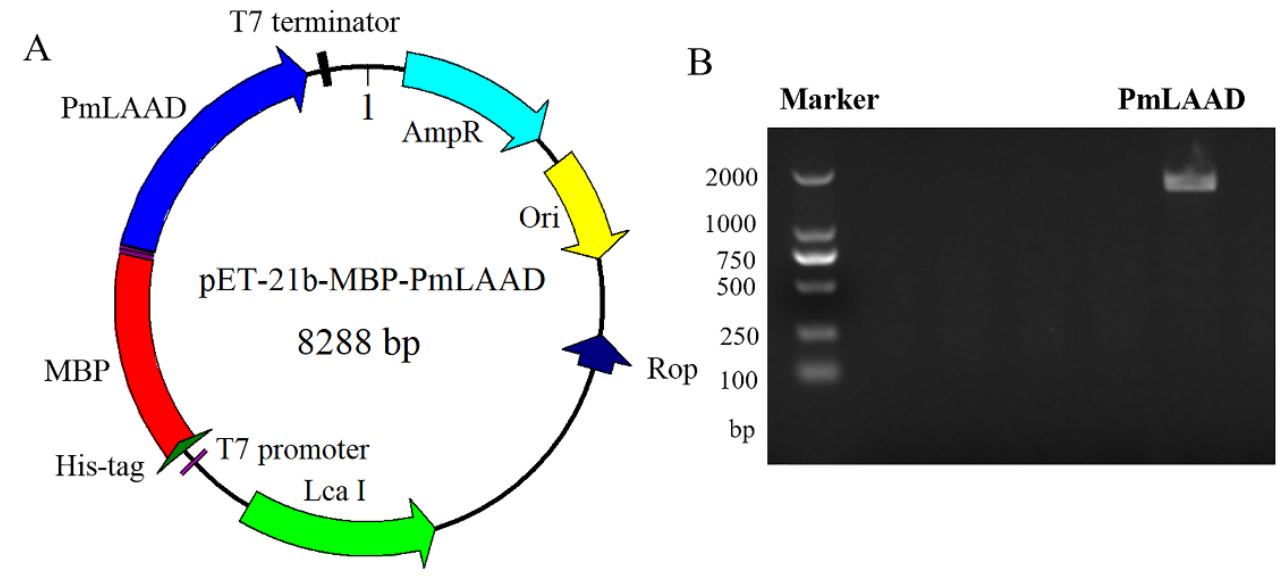

Figure 1. Construction of the recombinant plasmid of pET-21b-MBP-PmLAAD. (A) A schematic representation of the pET-21b-MBP-PmLAAD plasmid. The PmLAAD gene was inserted into the pET-21b-MBP vectors. (B) PCR amplification of the PmLAAD gene.

\subsection{Expression and Purification of MBP-PmLAAD}

The MBP-PmLAAD and PmLAAD expressions were induced at an $\mathrm{OD}_{600} \mathrm{~nm}$ of 0.8 with $0.5 \mathrm{mM}$ isopropyl $\beta$-D-1-thiogalactopyranoside (IPTG), at $17^{\circ} \mathrm{C}$ for $12 \mathrm{~h}$. As shown in Figure 2A, when the PmLAAD was not fused to the MBP-tag, the molecular weight of the PmLAAD was $52 \mathrm{kDa}$. Only a small portion of the PmLAAD was present in the soluble fraction and most of it was found in the membrane fraction. These results are in agreement with Baek et al., who expressed a second L-amino acid deaminase type named Pm1 from P. mirabilis KCTC 2566 and reported that most of the Pm1 protein was present in the membrane fraction [2]. The molecular weight of the MBP was $41 \mathrm{kDa}$, and when the PmLAAD was fused to the MBP-tag, the molecular weight of the corresponding MBP-PmLAAD product was $93 \mathrm{kDa}$ (Figure 2B). Lane 1 in Figure 2C also shows the protein band of purified MBP-PmLAAD, which is consistent with the MBP-PmLAAD protein band in Figure 2B. The SDS-PAGE showed that most of the MBP-PmLAAD protein was in the soluble fraction, thus the fusion to the MBP released the LAAD from the cell membranes and produced the soluble expression of the protein. Compared with previously reported methods of removing the N-terminal transmembrane region from the enzymes, such as the PmLAAD and PvLAAD which were still expressed in the form of an inclusion body [3,9], the fusion with the MBP-tag here could release the membrane binding state of the PmLAAD and promote its soluble expression.

The MBP-PmLAAD fusion protein was purified using affinity chromatography with a Ni-column; the results of the SDS-PAGE analyses are shown in Figure 2C. In order to better study the catalytic properties of the PmLAAD, an enzymatic digestion of the MBP-PmLAAD fusion with a rTEV protease followed by a purification of the reaction liquid with a Ni-column was used to remove the MBP-tag. The SDS-PAGE showed that once the MBP-tag was removed, the PmLAAD still remained a soluble protein. The molecular weight of the purified PmLAAD was $52 \mathrm{kDa}$.

To investigate the effect of the MBP fusion on the catalytic activity of PmLAAD, the activities of the PmLAAD and MBP-PmLAAD cell lysates, the MBP-PmLAAD fusion protein, and the purified PmLAAD were analyzed (Table 1). The purified MBP-PmLAAD fusion protein had a low activity of $0.025 \mu \mathrm{mol} / \mathrm{mg}$ PmLAAD-min using L-Phe as a substrate. The activity of the MBP-PmLAAD cell lysate was $0.17 \mu \mathrm{mol} / \mathrm{mg}$ PmLAAD·min, which was 2.2 times higher than the activity of the PmLAAD cell lysate. After removing the MBP-tag with the rTEV protease, the activity of the purified PmLAAD was not detected, which may be due to the instability of the PmLAAD without the fusion tag and/or membrane components. Since the fusion protein still has some activity, the catalytic properties of the fusion protein could be further investigated. 

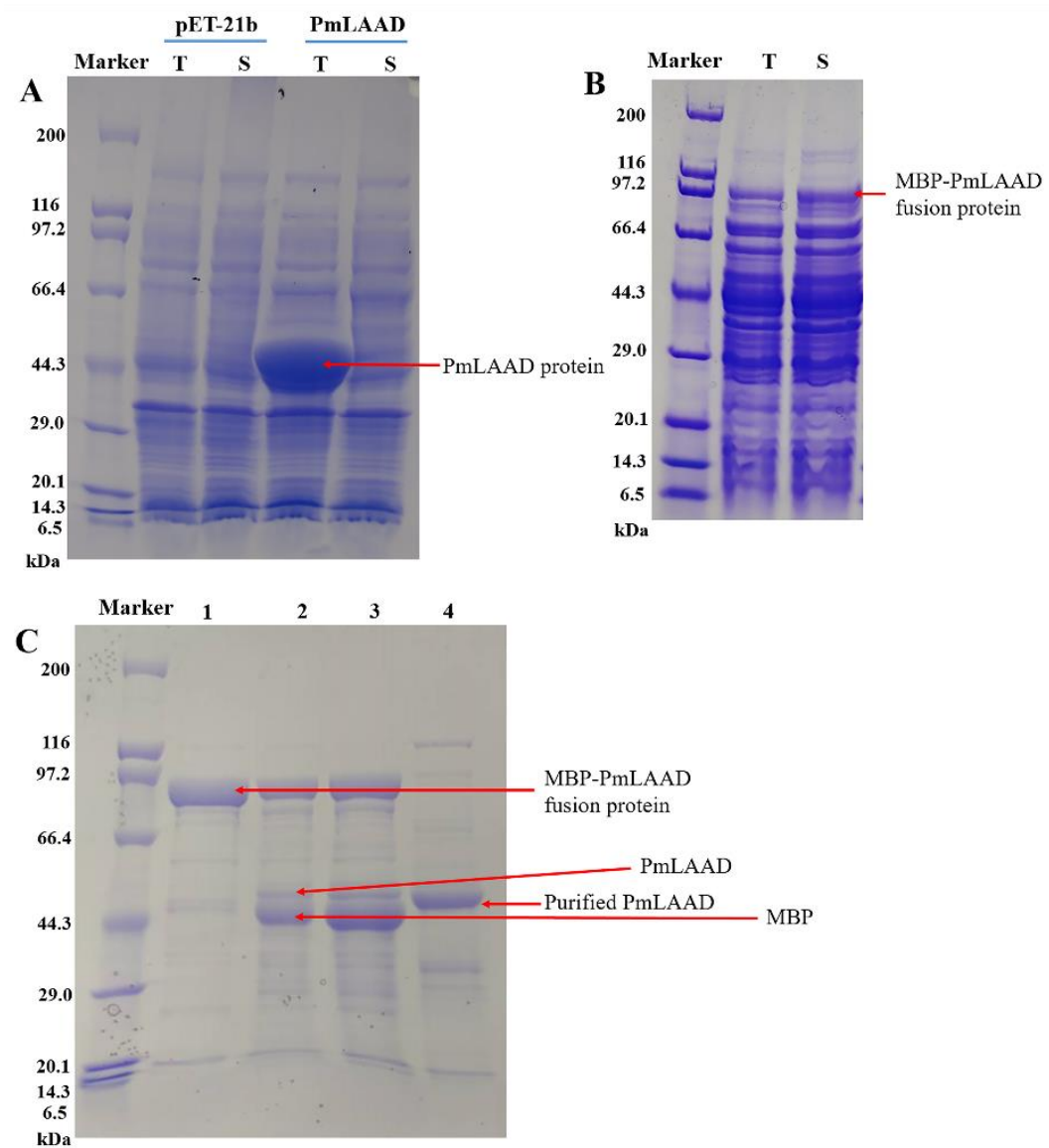

Figure 2. The SDS-PAGE of the PmLAAD and MBP-PmLAAD cell lysates and the purified MBP-PmLAAD. (A) E. coli BL21 (DE3)/pET-21b cell lysate (pET-21b), total cell lysate (T) and soluble fraction (S) of E. coli BL21 (DE3)/pET-21b-PmLAAD. The samples were generated from $50 \mathrm{mg} / \mathrm{mL}$ E. coli BL21 (DE3)/pET-21b cells and $50 \mathrm{mg} / \mathrm{mL}$ E. coli BL21 (DE3)/pET-21b-PmLAAD cells. (B) Total cell lysate (T) and soluble fraction (S) of E. coli BL21 (DE3)/pET-21b-MBP-PmLAAD. Lane T, E. coli BL21 (DE3)/pET-21b-MBP-PmLAAD cell lysate before centrifugation; lane S, E. coli BL21 (DE3)/pET-21b-MBP-PmLAAD cell lysate after centrifugation. The samples were generated from $50 \mathrm{mg} / \mathrm{mL}$ E. coli BL21 (DE3)/pET-21b-MBP-PmLAAD cells. (C) Purified and rTEV protease-treated MBP-PmLAAD fusion protein. Lane 1, proteins purified with Ni-column affinity chromatography; lane 2, rTEV protease-treated MBP-PmLAAD fusion protein, i.e., the mixture of MBP-PmLAAD, PmLAAD, and MBP; lane 3, the sample after removal of PmLAAD by secondary purification with a Ni-NTA column, i.e., the mixture of MBP-PmLAAD and MBP; lane 4, purified PmLAAD.

Table 1. Enzyme activities of the cell lysate, MBP-PmLAAD fusion protein, and PmLAAD a .

\begin{tabular}{cccccc}
\hline & $\begin{array}{c}\text { PmLAAD Cell } \\
\text { Lysate }\end{array}$ & $\begin{array}{c}\text { MBP-PmLAAD } \\
\text { Cell Lysate }\end{array}$ & $\begin{array}{c}\text { MBP-PmLAAD } \\
\text { Purified Enzyme }\end{array}$ & $\begin{array}{c}\text { PmLAAD Purified } \\
\text { Enzyme }\end{array}$ & $\begin{array}{c}\text { E. coli pET-21b } \\
\text { Cell Lysate }\end{array}$ \\
\hline $\begin{array}{c}\text { Specific activity } \\
(\mu \mathrm{mol} / \mathrm{mg}\end{array}$ & 0.076 & 0.17 & 0.025 & $\mathrm{ND}^{\mathrm{b}}$ & $\mathrm{ND}^{\mathrm{b}}$ \\
PmLAAD min $)$ & & & & & \\
\hline
\end{tabular}

${ }^{a}$ The reaction time was 30 min. ${ }^{b}$ Not detected.

\subsection{Biochemical Characterization of the MBP-PmLAAD Fusion Enzyme}

The effect of the $\mathrm{pH}$ and temperature on the MBP-PmLAAD fusion enzyme was investigated in order to study the catalytic properties of the enzyme. The range of temperatures at which the purified MBP-PmLAAD showed activity was 30 to $50{ }^{\circ} \mathrm{C}$. The enzyme activity increased as the temperature 
increased from 30 to $37^{\circ} \mathrm{C}$ and then decreased with a temperature increase from 37 to $50{ }^{\circ} \mathrm{C}$, reaching its maximum activity at $37^{\circ} \mathrm{C}$ (Figure $3 \mathrm{~A}$ ). The purified MBP-PmLAAD was active at $\mathrm{pH}$ ranging from 7 to 10 with its optimal activity at $\mathrm{pH} 8.0$ (Figure 3B). The activity of the MBP-PmLAAD decreased with a further increase in $\mathrm{pH}$ from 9 to 10. Subsequently, the stability of the MBP-PmLAAD was studied. As shown in Figure 3C, the MBP-PmLAAD fusion enzyme was relatively stable under the reaction conditions during the reaction for $10 \mathrm{~h}$, even after the reaction reached equilibrium.
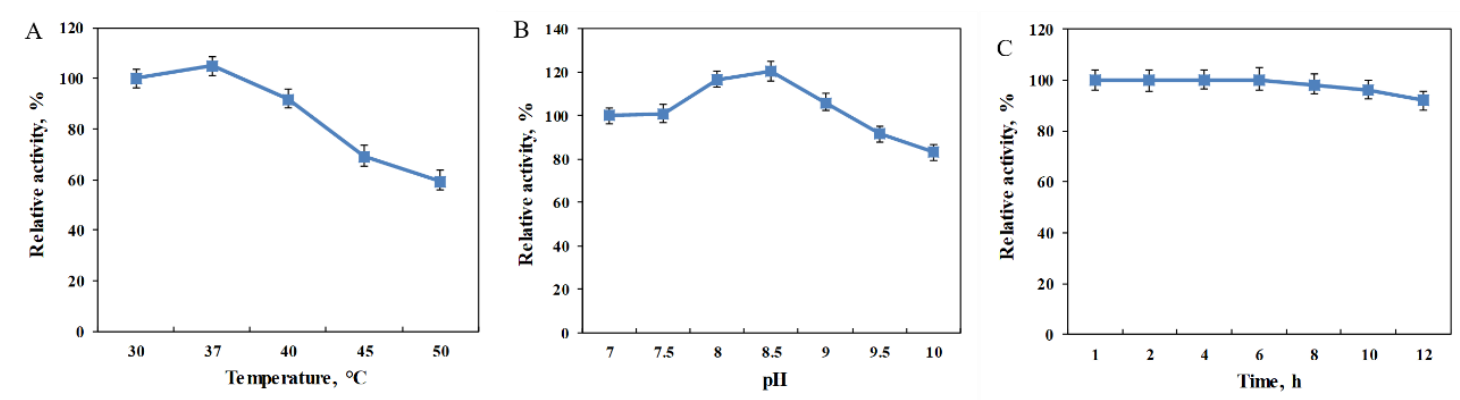

Figure 3. The effect of $\mathrm{pH}$, temperature and storage time on the activity of the purified MBP-PmLAAD fusion enzyme. (A) The effect of temperature on fusion enzyme activity. (B) The effect of $\mathrm{pH}$ on fusion enzyme activity. (C) The stability of the purified MBP-PmLAAD.

The results show that the catalytic efficiency of the purified enzyme was much lower than the catalytic efficiency of the crude enzyme solution (Table 1). Therefore, the effect of different catalyst concentrations on the catalytic efficiency was explored. Figure $4 \mathrm{~B}$ shows that for the same concentration, the amount of MBP-PmLAAD in the purified enzymes was much higher than in crude enzymes. However, the catalytic efficiency of the cell lysate with the same concentration was much higher than the catalytic efficiencyof the purified enzyme. As shown in Figure 4A, the conversion of $30 \mathrm{mM}$ L-Phe catalyzed by $4.25 \mathrm{mg} / \mathrm{mL}$ of the cell lysate (crude enzyme) was $100 \%$ in $2 \mathrm{~h}$, while the conversion of $30 \mathrm{mM}$ L-Phe catalyzed by $17 \mathrm{mg} / \mathrm{mL}$ of the pure enzyme was only $33 \%$ in $4 \mathrm{~h}$. Therefore, the activity of the MBP-PmLAAD cell lysate was much higher than the one of the purified MBP-PmLAAD fusion enzyme, although the content of the target protein in the pure enzyme suspension was higher. At the same time, the mixture of $3 \mathrm{mg} / \mathrm{mL}$ purified MBP-PmLAAD and $50 \mathrm{mg} / \mathrm{mL}$ E. coli BL21 (DE3)/pET-21b cell lysate was also used to catalyze $30 \mathrm{mM}$ L-Phe. As shown in Figure 4A, when the purified MBP-PmLAAD was mixed with the E. coli BL21 (DE3)/pET-21b cell lysate, $30 \mathrm{mM}$ of L-Phe was completely transformed to PPA in $2 \mathrm{~h}$. As the PmLAAD was the membrane-binding enzyme, the membrane components in the MBP-PmLAAD cell lysate could promote the catalytic reaction of MBP-PmLAAD. Similarly, it has been proposed that the membrane-bound LAADs are associated with the electron transport chain on the bacterial membrane and electrons are transferred to cytochrome oxidases to reduce $\mathrm{O}_{2}$ to $\mathrm{H}_{2} \mathrm{O}[9,12,31]$. This may be the reason why the activity of the MBP-PmLAAD cell lysate was higher than the activity of the purified enzyme. Therefore, by comparing the catalytic efficiency between the MBP-PmLAAD purified enzyme and the cell lysate, the membrane components in the cell lysate were found to promote the catalytic efficiency of the MBP-PmLAAD. Although the purified MBP-PmLAAD had low enzyme activity, considering the feasibility of the LAAD-catalyzed conversion of L-Phe to PPA, the fusion of the MBP-tag to the PmLAAD promoted the soluble active expression of the PmLAAD in the E. coli system, and the obtained cell lysate system was more favorable for enhanced catalytic performance. At the same time, the fusion expression of the MBP-tag to the PmLAAD enabled us to obtain the cell lysate system which can be used for catalysis. 

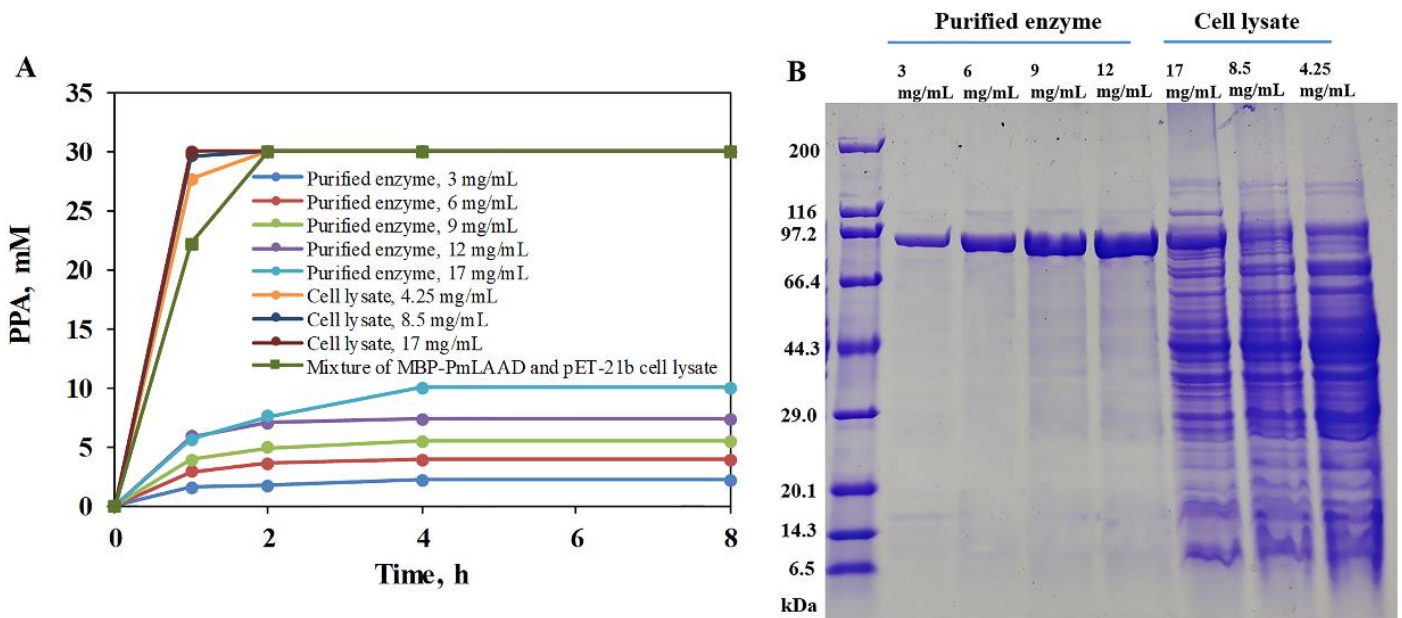

Figure 4. The effect of biocatalyst concentration and E. coli BL21 (DE3)/pET-21b cell lysate on the catalytic efficiency. (A) The catalytic conversion of L-Phe to PPA with different concentrations of the MBP-PmLAAD purified enzyme and cell lysate. The mixture of the MBP-PmLAAD and pET-21b cell lysate was composed of $3 \mathrm{mg} / \mathrm{mL}$ purified MBP-PmLAAD and $50 \mathrm{mg} / \mathrm{mL}$ E. coli BL21 (DE3)/pET-21b cell lysate. (B) The SDS-PAGE analysis of different concentrations of the MBP-PmLAAD purified enzyme and cell lysate. The reactions were all conducted in a $50 \mathrm{mM}, \mathrm{pH} 8.5$ Tris- $\mathrm{HCl}$ buffer at $37{ }^{\circ} \mathrm{C}$.

\subsection{Conversion of L-Phe to PPA with MBP-PmLAAD Cell Lysate}

In order to test the catalytic properties of the MBP-PmLAAD, the conversions of $50 \mathrm{mM}$ and $100 \mathrm{mM}$ L-Phe were catalyzed with the MBP-PmLAAD cell lysate and the PmLAAD whole-cell biocatalyst, respectively. As shown in Figure 5, when the catalyst was the MBP-PmLAAD cell lysate, $50 \mathrm{mM}$ of L-Phe was fully converted to PPA after $2 \mathrm{~h}$, while $100 \mathrm{mM}$ of L-Phe was fully converted to PPA in $6 \mathrm{~h}$. However, when $50 \mathrm{mM}$ of L-Phe was catalyzed with the PmLAAD whole cell, the conversion to PPA was $66.3 \%$. It is obvious that the catalytic efficiency of the PmLAAD whole cell was lower than the catalytic efficiency of the MBP-PmLAAD crude enzyme solution. Therefore, the MBP-tag allowed the soluble expression of the membrane-binding PmLAAD, and the soluble expression of the MBP-PmLAAD fusion protein would contribute to improving the catalytic activity of the membrane-binding PmLAAD.

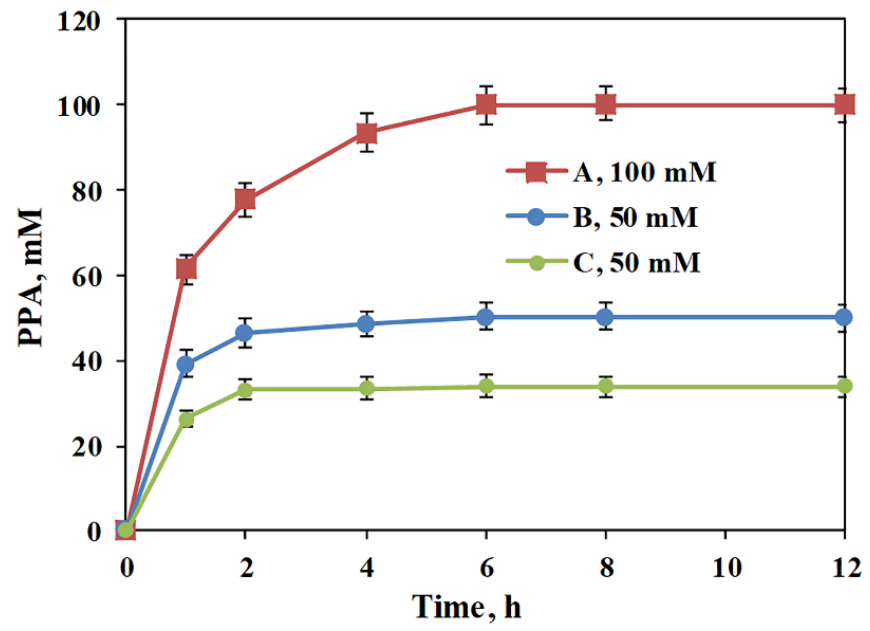

Figure 5. The time course of the formation of PPA with different biocatalysts. A: the biocatalyst was the MBP-PmLAAD cell lysate at $50 \mathrm{mg} / \mathrm{mL}$, with $100 \mathrm{mM}$ L-Phe; B: the biocatalyst was the MBP-PmLAAD cell lysate at $50 \mathrm{mg} / \mathrm{mL}$, with $50 \mathrm{mM}$ L-Phe; C: the biocatalyst was the PmLAAD whole cell at $100 \mathrm{mg} / \mathrm{mL}$, with $50 \mathrm{mM}$ L-Phe. The reactions were all conducted in a $50 \mathrm{mM}, \mathrm{pH} 8.5$ Tris- $\mathrm{HCl}$ buffer at $37^{\circ} \mathrm{C}$. 


\section{Materials and Methods}

\subsection{Materials}

The plasmid pET-21b-MBP and the PmLAAD gene from P. mirabilis (GenBank accession no. EU669819.1) were stored in our laboratory [2,4]. The enzymes, oligonucleotides, and other reagents for DNA cloning and amplification were obtained from the Takara-Bio Co., Kawasaki, Japan and the Novagen Co., Madison, WI, USA. The L-phenylalanine (L-Phe) and PPA were purchased from the Sinopharm Chemical Reagent Co., Ltd., Shanghai, China. The rTEV protease was purchased from Beijing Solarbio Science \& Technology Co., Ltd., Beijing, China. The Bradford Protein Assay Kit was purchased from Beijing Solarbio Science \& Technology Co., Ltd., Beijing, China.

\subsection{Construction of the Expression Plasmid pET-21b-MBP-PmLAAD}

The PmLAAD gene fragment flanked by the recognition sequences for Nde I and Sal I was inserted into the expression vector pET-21b-MBP, encoding an N-terminal maltose-binding protein, followed by a recognition site for the rTEV protease under the control of a T7 promoter. The primers used for plasmid construction were 5' -CTGTTTTTCAGAGCCATATGATGGCAATAAGTAGAAGAAAATT TATT-3' and 5'-ATCCTCGAGAAGCTTTGCGTCGACTTAGAAACGATACAGACTAAATGGT-3' . Then, the constructed plasmid pET-21b-MBP-PmLAAD was transformed into E. coli BL21 (DE3) cells. After plating, individual colonies were picked and the plasmids were sequenced.

\subsection{Expression and Purification of MBP-PmLAAD Fusion Proteins}

Recombinant E. coli BL21 (DE3) was grown in 1 L of Luria-Bertani medium, containing $100 \mathrm{mg} / \mathrm{mL}$ ampicillin at $37^{\circ} \mathrm{C}$. The culture was induced by the addition of isopropyl $\beta$-D-1-thiogalactopyranoside (IPTG) to a final concentration of $0.5 \mathrm{mM}$ when the optical density $(\lambda=600 \mathrm{~nm})$ was $0.6-0.8$. Furthermore, the culture was incubated for an additional $16 \mathrm{~h}$ at $17^{\circ} \mathrm{C}$ and $200 \mathrm{rpm}$. After centrifugation at $6000 \times g$ at $4{ }^{\circ} \mathrm{C}$ for $20 \mathrm{~min}$, the recombinant cells were washed with a potassium phosphate buffer.

To purify the MBP-PmLAAD, the recombinant E. coli BL21 (DE3) cells were resuspended in a lysis buffer containing $20 \mathrm{mM}$ Tris- $\mathrm{HCl}$ and $150 \mathrm{mM} \mathrm{NaCl}(\mathrm{pH}$ 7.5). The cell lysates were produced with a high-pressure homogenizer (APV, Lubeck, Germany) and then centrifuged at $12000 \times g$ for 30 min at $4{ }^{\circ} \mathrm{C}$ to remove cell debris. The proteins were purified by Ni affinity chromatography with an $\ddot{A} k t a$ Purifier 10 (GE Healthcare, Pittsburgh, PA, USA). The gradient elution buffer for the Ni-NTA column was composed of $20 \mathrm{mM}$ Tris- $\mathrm{HCl}$ (pH 7.5), $150 \mathrm{mM} \mathrm{NaCl}$, and 0-500 mM imidazole. The imidazole in the protein solution was removed using a PD-10 desalting column (GE Healthcare, USA). The MBP-tag was removed with the rTEV protease by adding $1000 \mu \mathrm{g}$ of MBP-PmLAAD fusion protein and $10 \mu \mathrm{g}$ of rTEV Protease to $1000 \mu \mathrm{L}$ of buffer $\left(50 \mathrm{mM} \mathrm{NaH}_{2} \mathrm{PO}_{4}, 150 \mathrm{mM} \mathrm{NaCl}, 1 \mathrm{mM}\right.$ EDTA, $1 \mathrm{mM}$ DTT, $10 \%$ glycerol, $\mathrm{pH} 8.0$ ), then the reaction mixture was kept at $16^{\circ} \mathrm{C}$ for $6 \mathrm{~h}$. Subsequently, the MBP-tag and rTEV protease were removed with a Ni-NTA column. The protein concentrations were determined using a Bradford Protein Assay Kit (Solarbio Science \& Technology, Beijing, China).

\subsection{Activity Assay}

The PmLAAD and MBP-PmLAAD cell lysates, the purified PmLAAD enzyme, and the MBP-PmLAAD purified enzyme were incubated in $50 \mathrm{mM}$ Tris-HCl buffer ( $\mathrm{pH} 8.0)$ with $30 \mathrm{mM}$ L-Phe in a final volume of $5.0 \mathrm{~mL}$ at $37^{\circ} \mathrm{C}$. After reaction for $30 \mathrm{~min}$, an equal volume of $10 \%(w / v)$ trichloroacetic acid solution was added to stop the reaction. The mixture was centrifuged at $12000 \times g$ for $5 \mathrm{~min}$, and a $15 \mu \mathrm{L}$ aliquot of the supernatant was added to $1 \mathrm{~mL}$ of ferric chloride solution and incubated for $2 \mathrm{~min}$ at room temperature. The concentration of the PPA was determined by measuring the $\mathrm{OD}_{640 \mathrm{~nm}}$. One PmLAAD unit corresponds to the amount of enzyme that generates $1 \mu \mathrm{mol}$ of PPA product per minute [6]. 


\subsection{Effect of $p H$, Temperature and Storage Time on the Activity of MBP-PmLAAD}

To investigate the effect of the reaction temperature on the MBP-PmLAAD catalyzing oxidative deamination of L-Phe, $30 \mathrm{mM}$ L-Phe and $3.1 \mathrm{mg} / \mathrm{mL}$ MBP-PmLAAD were added to $2 \mathrm{~mL}$ Tris-HCl buffer $(50 \mathrm{mM}, \mathrm{pH} 7.5)$, and the reaction was conducted at $220 \mathrm{rpm}$ and different temperatures $\left(30^{\circ} \mathrm{C}\right.$, $37^{\circ} \mathrm{C}, 40^{\circ} \mathrm{C}, 45^{\circ} \mathrm{C}$, and $50^{\circ} \mathrm{C}$ ) for $30 \mathrm{~min}$. The effect of the initial $\mathrm{pH}$ on the MBP-PmLAAD catalyzing oxidative deamination of L-Phe was studied using the same mixture of L-Phe and MBP-PmLAAD described above and added to $2 \mathrm{~mL} 50 \mathrm{mM}$ Tris- $\mathrm{HCl}$ buffer with a $\mathrm{pH}$ varying from 7 to 10 ; the reaction was conducted at $220 \mathrm{rpm}$ and $37^{\circ} \mathrm{C}$ for $30 \mathrm{~min}$. To investigate the stability of the MBP-PmLAAD, the MBP-PmLAAD was stored in a Tris- $\mathrm{HCl}$ buffer $(50 \mathrm{mM}, \mathrm{pH} 8.5)$ at $37^{\circ} \mathrm{C}$ for $1-12 \mathrm{~h}$. Then, the activity of the MBP-PmLAAD was determined. The reaction buffer was a Tris- $\mathrm{HCl}$ buffer $(50 \mathrm{mM}$, $\mathrm{pH}$ 8.5), the L-Phe was $30 \mathrm{mM}$, and the reaction was conducted at $220 \mathrm{rpm}$ and $37^{\circ} \mathrm{C}$ for $30 \mathrm{~min}$. The PPA generated in the reaction process was measured using ferric chloride solution.

\subsection{Conversion of L-Phe to PPA Using the MBP-PmLAAD Fusion Protein}

The effect of the biocatalyst concentration on catalytic efficiency was assessed using different concentrations of the MBP-PmLAAD cell lysate $(4.25-17 \mathrm{mg} / \mathrm{mL})$ and the purified enzyme $(3-17 \mathrm{mg} / \mathrm{mL})$ that were added to the $2 \mathrm{~mL}, 50 \mathrm{mM}, \mathrm{pH} 8.5$ Tris- $\mathrm{HCl}$ buffer; the L-Phe concentration was $30 \mathrm{mM}$. The effect of the E. coli BL21 (DE3)/pET-21b cell lysate on the catalytic efficiency was assessed using the mixture of $3 \mathrm{mg} / \mathrm{mL}$ purified MBP-PmLAAD and $50 \mathrm{mg} / \mathrm{mL}$ E. coli BL21 (DE3)/pET-21b cell lysate, that were added to the $2 \mathrm{~mL}, 50 \mathrm{mM}, \mathrm{pH} 8.5$ Tris- $\mathrm{HCl}$ buffer; the L-Phe concentration was $30 \mathrm{mM}$. The reaction was conducted at $220 \mathrm{rpm}$ and $37^{\circ} \mathrm{C}$ and was stopped by adding an equal volume of $10 \%(w / v)$ trichloroacetic acid solution. The amount of PPA generated in the reaction was determined using ferric chloride solution. To compare the catalytic efficiency of the MBP-PmLAAD cell lysate and the PmLAAD whole-cell catalyst for catalyzing the transformation of L-Phe, $50 \mathrm{mg} / \mathrm{mL}$ of the MBP-PmLAAD whole-cell lysate or $100 \mathrm{mg} / \mathrm{mL}$ of the PmLAAD whole-cell biocatalyst was added to the $2 \mathrm{~mL}, 50 \mathrm{mM}$, pH 8.5 Tris- $\mathrm{HCl}$ buffer. The L-Phe concentrations were 50 and $100 \mathrm{mM}$, respectively. The reaction was conducted at $220 \mathrm{rpm}$ and $37^{\circ} \mathrm{C}$ and was stopped by adding an equal volume of $10 \%$ $(w / v)$ trichloroacetic acid solution. The amount of PPA generated in the reaction was determined using ferric chloride solution.

\section{Conclusions}

In summary, the membrane-bound protein PmLAAD was successfully expressed as a soluble active protein and therefore lost its membrane-binding status. The key factor in this study was the usage of an MBP-fusion protein as a solubility tag. Then, the MBP-PmLAAD fusion protein was purified and the purified soluble PmLAAD was obtained after the rTEV protease digestion. The activities of the MBP-PmLAAD cell lysate, purified MBP-PmLAAD, and purified PmLAAD were determined. The purified MBP-PmLAAD fusion protein showed a low activity with L-Phe, while the purified PmLAAD lost its activity after the rTEV protease digestion. At the same time, the activity of the MBP-PmLAAD cell lysate was much higher than that of the purified MBP-PmLAAD. By comparing the catalytic process of the MBP-PmLAAD cell lysate and the PmLAAD whole cells, we found that fusion with MBP could improve the active expression of PmLAAD and thus enhance catalytic performance. L-Phe at $100 \mathrm{mM}$ could be fully converted to PPA in $6 \mathrm{~h}$ by $50 \mathrm{mg} / \mathrm{mL}$ of MBP-PmLAAD-involved cell lysate. Therefore, the fusion expression of an MBP-tag to PmLAAD enables us to obtain a cell lysate system which can be used for highly efficient biocatalysis. In addition, this strategy provides a feasible way of improving the soluble expression of membrane-bound LAADs, which would be helpful in promoting the study of catalytic mechanisms of the enzyme.

Author Contributions: Conceptualization, D.-P.Z., Y.N. and Y.X.; investigation, D.-P.Z., X.-R.J., A.-W.F. and H.L.; formal analysis, D.-P.Z. and X.-R.J.; validation, X.-R.J., A.-W.F. and H.L.; writing-original draft preparation, D.-P.Z.; writing-review and editing, Y.N.; supervision, Y.N. and Y.X.; funding acquisition, Y.N. All authors have read and agreed to the published version of the manuscript. 
Funding: This work was financially supported by the National Natural Science Foundation of China [grant numbers 21676120, 31872891], the Program of Introducing Talents of Discipline to Universities [grant number 111-2-06], the High-End Foreign Experts Recruitment Program [grant number G20190010083], the Program for Advanced Talents within Six Industries of Jiangsu Province [grant number 2015-NY-007], the National Program for Support of Top-notch Young Professionals, Postgraduate Research \& Practice Innovation Program of Jiangsu Province [grant number KYCX18_1794], the Fundamental Research Funds for the Central Universities [grant number JUSRP51504], the Project Funded by the Priority Academic Program Development of Jiangsu Higher Education Institutions, Top-notch Academic Programs Project of Jiangsu Higher Education Institutions, the Jiangsu province "Collaborative Innovation Center for Advanced Industrial Fermentation" industry development program, and the National First-Class Discipline Program of Light Industry Technology and Engineering [grant number LITE2018-09].

Acknowledgments: We would like to thank Editage (www.editage.cn) for English language editing.

Conflicts of Interest: The authors declare no conflict of interest.

\section{References}

1. Pantaleone, D.P.; Geller, A.M.; Taylor, P.P. Purification and characterization of an L-amino acid deaminase used to prepare unnatural amino acids. J. Mol. Catal. B Enzym. 2001, 11, 795-803. [CrossRef]

2. Baek, J.O.; Seo, J.W.; Kwon, O.; Seong, S.I.; Kim, I.H.; Kim, C.H. Expression and characterization of a second L-amino acid deaminase isolated from Proteus mirabilis in Escherichia coli. J. Basic Microbiol. 2011, 51, 129-135. [CrossRef]

3. Liu, L.; Hossain, G.S.; Shin, H.D.; Li, J.H.; Du, G.C.; Chen, J. One-step production of $\alpha$-ketoglutaric acid from glutamic acid with an engineered L-amino acid deaminase from Proteus mirabilis. J. Biotechnol. 2013, 164, 97-104. [CrossRef]

4. Zhang, D.P.; Jing, X.R.; Zhang, W.L.; Nie, Y.; Xu, Y. Highly selective synthesis of D-amino acids from readily available L-amino acids by a one-pot biocatalytic stereoinversion cascade. RSC Adv. 2019, 9, 29927-29935. [CrossRef]

5. Hou, Y.; Hossain, G.S.; Li, J.H.; Shin, H.D.; Du, G.C.; Liu, L. Combination of phenylpyruvic acid (PPA) pathway engineering and molecular engineering of L-amino acid deaminase improves PPA production with an Escherichia coli whole-cell biocatalyst. Appl. Microbiol. Biotechnol. 2016, 100, 2183-2191. [CrossRef] [PubMed]

6. Hou, Y.; Hossain, G.S.; Li, J.; Shin, H.D.; Liu, L.; Du, G.C. Production of phenylpyruvic acid from L-phenylalanine using an L-amino acid deaminase from Proteus mirabilis: Comparison of enzymatic and whole-cell biotransformation approaches. Appl. Microbiol. Biotechnol. 2015, 99, 8391-8402. [CrossRef] [PubMed]

7. Song, Y.; Li, J.; Shin, H.D.; Du, G.C.; Liu, L.; Chen, J. One-step biosynthesis of $\alpha$-ketoisocaproate from L-leucine by an Escherichia coli whole-cell biocatalyst expressing an L-amino acid deaminase from Proteus vulgaris. Sci. Rep. 2015, 5, 12614-12625. [CrossRef] [PubMed]

8. Molla, G.; Melis, R.; Pollegioni, L. Breaking the mirror: L-Amino acid deaminase, a novel stereoselective biocatalyst. Biotechnol. Adv. 2017, 35, 657-668. [CrossRef]

9. Ju, Y.; Tong, S.; Gao, Y.; Zhao, W.; Liu, Q.; Gu, Q.; Xu, J.; Niu, L.; Teng, M.; Zhou, H. Crystal structure of a membrane-bound L-amino acid deaminase from Proteus vulgaris. J. Struct. Biol. 2016, 195, $306-315$. [CrossRef]

10. Motta, P.; Molla, G.; Pollegioni, L.; Nardini, M. Structure-function relationships in L-amino acid deaminase, a flavoprotein belonging to a novel class of biotechnologically relevant enzymes. J. Biol. Chem. 2016, 291, 10457-10475. [CrossRef]

11. Hossain, G.S.; Li, J.; Shin, H.D.; Du, G.C.; Wang, M.; Liu, L.; Chen, J. One-step biosynthesis of $\alpha$-keto- $\gamma$-methylthiobutyric acid from L-methionine by an Escherichia coli whole-cell biocatalyst expressing an engineered L-amino acid deaminase from Proteus vulgaris. PLoS ONE 2014, 9, 114291-114307. [CrossRef] [PubMed]

12. Hossain, G.S.; Li, J.; Shin, H.D.; Chen, R.R.; Du, G.C.; Liu, L.; Chen, J. Bioconversion of L-glutamic acid to $\alpha$-ketoglutaric acid by an immobilized whole-cell biocatalyst expressing L-amino acid deaminase from Proteus mirabilis. J. Biotechnol. 2014, 169, 112-120. [CrossRef] [PubMed] 
13. Nshimiyimana, P.; Liu, L.; Du, G.C. Engineering of L-amino acid deaminases for the production of $\alpha$-keto acids from L-amino acids. Bioengineered 2019, 10, 43-51. [CrossRef] [PubMed]

14. Hossain, G.S.; Shin, H.D.; Li, J.H.; Du, G.C.; Chen, J.; Liu, L. Transporter engineering and enzyme evolution for pyruvate production from $\mathrm{d} / \mathrm{L}$-alanine with a whole-cell biocatalyst expressing $\mathrm{L}$-amino acid deaminase from Proteus mirabilis. RSC Adv. 2016, 6, 82676-82684. [CrossRef]

15. Hossain, G.S.; Shin, H.D.; Li, J.; Wang, M.; Du, G.C.; Liu, L.; Chen, J. Integrating error-prone PCR and DNA shuffling as an effective molecular evolution strategy for the production of $\alpha$-ketoglutaric acid by L-amino acid deaminase. RSC Adv. 2016, 6, 46149-46158. [CrossRef]

16. Li, R.; Sakir, H.G.; Li, J.; Shin, H.D.; Du, G.C.; Chen, J.; Liu, L. Rational molecular engineering of L-amino acid deaminase for production of $\alpha$-ketoisovaleric acid from L-valine by Escherichia coli. RSC Adv. 2017, 7, 6615-6621. [CrossRef]

17. Okino, S.; Inui, M.; Yukawa, H. Production of organic acids by Corynebacterium glutamicum under oxygen deprivation. Appl. Microbiol. Biotechnol. 2005, 68, 475-480. [CrossRef]

18. Hanson, R.L.; Davis, B.L.; Goldberg, S.L.; Johnston, R.M.; Parker, W.L.; Tully, T.P.; Montana, M.A.; Patel, R.N. Enzymatic preparation of a D-amino acid from a racemic amino acid or keto acid. Org. Process. Res. Dev. 2008, 12, 1119-1129. [CrossRef]

19. Parmeggiani, F.; Lovelock, S.L.; Weise, N.J.; Ahmed, S.T.; Turner, N.J. Synthesis of D- and L-phenylalanine derivatives by phenylalanine ammonia lyases: A multienzymatic cascade process. Angew. Chem. Int. Ed. 2015, 54, 4608-4611. [CrossRef]

20. Busto, E.; Richter, N.; Grischek, B.; Kroutil, W. Biocontrolled formal inversion or retention of L- $\alpha$-amino acids to enantiopure (R)- or (S)-hydroxyacids. Chem. Eur. J. 2014, 20, 11225-11228. [CrossRef]

21. Parmeggiani, F.; Ahmed, S.T.; Thompson, M.P.; Weise, N.J.; Galman, J.L.; Gahloth, D.; Dunstan, M.S.; Leys, D.; Turner, N.J. Single-biocatalyst synthesis of enantiopured-arylalanines exploiting an engineered D-amino acid dehydrogenase. Adv. Synth. Catal. 2016, 358, 3298-3306. [CrossRef]

22. Zhao, W.; Ding, H.; Lv, C.; Hu, S.; Huang, J.; Zheng, X.; Yao, S.; Mei, L. Two-step biocatalytic reaction using recombinant Escherichia coli cells for efficient production of phenyllactic acid from L-phenylalanine. Process Biochem. 2018, 64, 31-37. [CrossRef]

23. Zhu, L.; Feng, G.; Ge, F.; Song, P.; Wang, T.; Liu, Y.; Tao, Y.; Zhou, Z. One-pot enzymatic synthesis of D-arylalanines using phenylalanine ammonia lyase and L-amino acid deaminase. Appl. Biochem. Biotechnol. 2018, 187, 75-89. [CrossRef] [PubMed]

24. Melis, R.; Rosini, E.; Pirillo, V.; Pollegioni, L.; Molla, G. In vitro evolution of an L-amino acid deaminase active on L-1-naphthylalanine. Catal. Sci. Technol. 2018, 8, 5359-5367. [CrossRef]

25. Korepanova, A.; Moore, J.D.; Nguyen, H.B.; Hua, Y.; Cross, T.A.; Gao, F. Expression of membrane proteins from Mycobacterium tuberculosis in Escherichia coli as fusions with maltose binding protein. Prot. Expr. Purif. 2007, 53, 24-30. [CrossRef]

26. Cabanne, C.; Pezzini, J.; Joucla, G.; Hocquellet, A.; Barbot, C.; Garbay, B.; Santarelli, X. Efficient purification of recombinant proteins fused to maltose-binding protein by mixed-mode chromatography. J. Chromatogr. A 2009, 1216, 4451-4456. [CrossRef]

27. Bokhove, M.; Sadat Al Hosseini, H.; Saito, T.; Dioguardi, E.; Gegenschatz-Schmid, K.; Nishimura, K.; Raj, I.; de Sanctis, D.; Han, L.; Jovine, L. Easy mammalian expression and crystallography of maltose-binding protein-fused human proteins. J. Struct. Biol. 2016, 194, 1-7. [CrossRef]

28. Moua, P.S.; Gonzalez, A.; Oshiro, K.T.; Tam, V.; Li, Z.H.; Chang, J.; Leung, W.; Yon, A.; Thor, D.; Venkatram, S.; et al. Differential secretion pathways of proteins fused to the Escherichia coli maltose binding protein (MBP) in Pichia pastoris. Prot. Expr. Purif. 2016, 124, 1-9. [CrossRef]

29. Hahn, K.; Neumeister, K.; Mix, A.; Kottke, T.; Groger, H.; Fischer von Mollard, G. Recombinant expression and characterization of a L-amino acid oxidase from the fungus Rhizoctonia solani. Appl. Microbiol. Biotechnol. 2017, 101, 2853-2864. [CrossRef] 
30. Guo, Y.; Yu, M.; Jing, N.; Zhang, S. Production of soluble bioactive mouse leukemia inhibitory factor from Escherichia coli using MBP tag. Prot. Expr. Purif. 2018, 150, 86-91. [CrossRef]

31. Ju, Y.; Liu, Z.; Zhang, Z.; Duan, L.; Liu, Q.; Gu, Q.; Zhang, C.; Xu, J.; Zhou, H. Membrane binding of the insertion sequence of Proteus vulgaris L-amino acid deaminase stabilizes protein structure and increases catalytic activity. Sci. Rep. 2017, 7, 13719-13730. [CrossRef] [PubMed] 